\title{
Luminosity Function of Arakelian Galaxies and Their Environmental Dependences
}

\author{
Abraham P. Mahtessian, Vardan G. Movsisyan, Lazar A. Mahtessian, Garen S. Karapetian \\ NAS RA V. Ambartsumian Byurakan Astrophysical Observatory (BAO), Armenia \\ Email: amahtes@bao.sci.am
}

\begin{abstract}
This article proposes a method for constructing the luminosity function of galaxies, which gives a result that is consistent with other methods, is as accurate as other methods, and is simple. Using this method, we constructed the luminosity function of Arakelian galaxies with different morphological types in different environments, included in the CfA2 catalog. The proposed method of identifying groups of galaxies is physically grounded and simple. It was found that both the bright and weak parts of the normalized luminosity function of Arakelian galaxies are higher than the bright and weak parts of the similar function of the total sample CfA2. A similar phenomenon is observed separately for members of groups, as well as for single galaxies. This also applies to small groups with 2 or 3 galaxies, and more populated groups. Spiral galaxies obey these laws. For elliptical galaxies, the picture is not very clear (due to the small number of well-known elliptical Arakelian galaxies). The spatial density of the number of ArG in the $\mathrm{M} \leqslant-17$ range is $0.00068 \pm 0.00005 \mathrm{Mpc}^{\wedge}-3$, which corresponds to $2.7 \%$ of the entire sample in this range (for CfA2 $\rho(\mathrm{M} \leqslant-17)=0.0254 \pm 0.0002 \mathrm{Mpc}^{\wedge}$ $3)$.
\end{abstract}

Keywords: Galaxies, groups of galaxies, Arakelian galaxies, luminosity function, environment

\section{Introduction}

The interaction of galaxies with their environment can lead to a change in their luminosities and, ultimately, to a change in the distribution of luminosities of galaxies.

In order to study the variation in the integral characteristics of galaxies and the occurrence of peculiarity in their nuclei, Arakelian [1] compiled a list of galaxies with high surface brightnesses. The surface brightnesses of the galaxies were calculated using the formula

$$
\tilde{B}=m-0.25 \cos e c\left|b^{I I}\right|+2.5 \lg \frac{\pi a b}{4}+0.22(a / b)+0.73
$$

where $\tilde{B}$ is the average surface brightness of a galaxy, $m$ is its apparent magnitude [2], $a$ and $b$ are the external dimensions of a galaxy [3], and the last two terms are introduced to take into account the inclination of the galaxy and roughly approximate the surface brightness of the galaxy to Holmberg's system. Galaxies with $\tilde{B} \leq 22.0$ were chosen as galaxies with a high surface brightness. They constitute about $4 \%$ of all CGCG galaxies.

In [4], the connection between Arakelian galaxies of high surface brightness and their environment was studied. In particular, it has been found that Arakelian galaxies (ArG) which are members of groups have higher luminosities than single ones on average.

The luminosity function (LF) of the ArG and their spatial density were previously studied in [5]. The increase in observational data in the past time and the creation of new catalogues of groups of galaxies will allow us to study the dependence of the LF of the ArG of various morphological types on the environment.

\section{The Method of Constructing the Luminosity Function of Galaxies}

To determine the luminosity function of galaxies, we will use the method proposed in [6].

In general, in [6], the Schmidt method [7] was generalized in order to take into account the dependence of the density of galaxies on the distance in the nearby Universe. The LF is defined by the following formula: 


$$
\Phi_{o b s}\left(M_{i}\right)=\left\{\begin{array}{lc}
\frac{1}{\Delta M_{M_{i} \pm \Delta M / 2, j} \frac{1}{D\left(r_{m}^{j}\right)\left(V_{m}^{j}-V_{\min }\right)},} & M_{\max } \geq M_{i} \geq M_{\min } \\
\frac{1}{\Delta M D\left(r_{\max }\right)\left(V_{\max }-V_{\min }\right)} \sum_{M_{i} \pm \Delta M / 2, j} 1 & M_{i}<M_{\min }
\end{array}\right.
$$

where $r_{m}^{i}=\frac{c z_{m}^{i}}{H}=\left(\frac{3 V_{m}^{i}}{\Omega}\right)^{1 / 3}$ - is the distance corresponding to the volume $V_{m}^{i} \cdot H=100 \mathrm{~km} \cdot \mathrm{s}^{-1} \cdot \mathrm{Mpc}^{-1}$ is the Hubble constant. Factually $D\left(r_{m}^{i}\right)$ - is the density of galaxies, normalized for the volume $V_{\max }$ : $D\left(r_{\max }\right)=1 . V_{m}^{i}=\frac{\Omega}{3}\left(\frac{c z_{m}^{i}}{H}\right)^{3}$ is the volume at the boundary of which a galaxy with an absolute stellar magnitude $M_{i}$ will have the maximum apparent magnitude of the sample $m_{\lim } ; V_{\min }=\frac{\Omega}{3}\left(\frac{c z_{\min }}{H}\right)^{3}$ is a close volume excluded from consideration, and $V_{\max }=\frac{\Omega}{3}\left(\frac{c z_{\max }}{H}\right)^{3}$ is the maximum volume, beyond which the galaxies are also not considered. $M_{i}=m_{i}-25-5 \log \left(c z_{i} / H\right), \quad M_{i}=m_{\lim }-25-5 \log \left(c z_{m}^{i} / H\right)$, $M_{\text {min }}=m_{\lim }-25-5 \log \left(c z_{\max } / H\right), \quad M_{\max }=m_{\lim }-25-5 \log \left(c z_{\min } / H\right), \Omega-$ is the solid sampling angle, and in our case equal to 4.3 .

RMS deviation $\Phi\left(M_{i}\right)$ is estimated as follows:

$$
\sigma\left(\Phi_{o b s}\left(M_{i}\right)\right)=\frac{1}{\Delta M D\left(r_{m}^{i}\right)\left(V_{m}^{i}-V_{\min }\right)}\left[n_{i}\left(1-\frac{n_{i}}{N}\right)\right]^{1 / 2}=\frac{\Phi_{o b s}\left(M_{i}\right)}{n_{i}}\left[n_{i}\left(1-\frac{n_{i}}{N}\right)\right]^{1 / 2}
$$

where $n_{i}$ - is the number of galaxies in the interval $M_{i} \pm \Delta M / 2, N$ - is the total number of galaxies in the sample.

These formulas will give the true density of the number of galaxies only when dealing with a full sample. When the sample is incomplete, and the completeness factor does not depend on the absolute magnitude, we can accurately estimate only the normalized LF of galaxies (for example, [8, 9]).

$$
\Psi\left(M_{i}\right)=\frac{\Phi_{o b s}\left(M_{i}\right)}{\sum_{j} \Phi_{o b s}\left(M_{j}\right)}
$$

The true density of the number of galaxies with an absolute value $M_{i}$ will be:

$$
\Phi\left(M_{i}\right)=P\left(m_{\lim }\right)^{-1} \Phi_{o b s}\left(M_{i}\right),
$$

and the RMS deviation will be:

$$
\sigma\left(\Phi\left(M_{i}\right)\right)=P\left(m_{\lim }\right)^{-1} \sigma\left(\Phi_{o b s}\left(M_{i}\right)\right)
$$

where $P\left(m_{\text {lim }}\right)$ is the completeness factor.

To study the completeness of the sample, limited by star magnitude, the $V / V_{m}$ method [7] is widely used, where $V$ is the volume of space on the edge of which the galaxy, and $V_{m}$ is the maximum volume of space, on the edge of which the galaxy will have an apparent magnitude equal to the maximum magnitude of the sample. If objects are evenly distributed in Euclidean space, then the average value of $<V / V_{m}>$ should equal 0.5 . In this space the distribution value $<V / V_{m}>$ is strictly equivalent to the distribution of visible values [9], which, with a uniform distribution of objects, will be $N(m) \sim 10^{0.6 m}$. It is assumed that the density of galaxies depends on the distance, and therefore this method is used for an approximate estimate of the completeness of the samples [6].

The dependence of the density of galaxies on the distance $D\left(r_{m}^{i}\right)$ can be constructed with the help of samples complete at the apparent and absolute magnitude. For this, three subsamples of galaxies were created, based on absolute magnitude (see [6]):

a) A sample with $M \leq-21^{m}$, which is complete in absolute magnitude in the entire volume studied. According to this sample, the desired dependence was determined at redshifts from $7000 \mathrm{~km} / \mathrm{s}$ to 20,000 $\mathrm{km} / \mathrm{s}$. At small distances, this sample is unsuitable due to the small number of galaxies. 
b) A sample with $M \leq-20^{m}$. This sample is complete until redshift of $12600 \mathrm{~km} / \mathrm{s}$. For this sample, the desired dependence is determined at redshifts from $1700 \mathrm{~km} / \mathrm{s}$ to $12600 \mathrm{~km} / \mathrm{s}$.

c) A sample with $M \leq-17.8^{m}$. For this sample, the desired dependence is determined for redshifts from $500 \mathrm{~km} / \mathrm{s}$ to $5000 \mathrm{~km} / \mathrm{s}$.

These dependences are merged in common areas and normalized to a redshift of $20,000 \mathrm{~km} / \mathrm{s}$. In [10], this problem was solved somewhat differently.

\section{Comparison of Methods for the Construction of LF Galaxies}

In recent decades, many methods have been proposed for constructing the luminosity function (LF) of galaxies. A detailed description of most of them can be found in the works of Felten [11] and Binggeli [12]. The $1 / V_{m}$ method, which was proposed by Schmidt [13] for studying the evolution of quasars, is very popular. Later it was used in [14] to estimate the LF of galaxies. In the $1 / V_{m}$ method, it is assumed that galaxies are evenly distributed in space. But it is well known that galaxies in the Universe are not evenly distributed. This leads to the problem that when applying the $1 / V_{m}$ method, we overestimate the relative number of faint galaxies.

In order to overcome the dependence on the assumption of a uniform distribution, Linden-Bell [15] proposed the so-called $C^{-}$method. He applied this method to a sample of quasars studied by Schmidt [13]. Linden-Bell testified that $C^{-}$estimation is a maximum likelihood method, and it does not require any assumptions about the distribution of galaxies. There is only one assumption that the LF in the direction of the line of sight at all points has the same appearance, and the sample must be ordered by luminosity. Subsequently, this method was generalized in [16] to consider several samples, for each of which the selection function was taken into account. Assuming also that the distribution of objects can be described by an analytic function, an error estimate was also obtained in [16].

In [17], a method was proposed similar to $C^{-}$. In this method, in contrast to [15], the authors divide the values of absolute magnitudes into intervals. The $C^{-}$method was first used for galaxies by Cholonevsky in [18]. The $C^{-}$method can give a correctly normalized LF, making no assumptions for the shape of the density distribution or luminosity of galaxies.

In [19] and [20], independently of each other, a method was proposed that builds the LF of galaxies, but does not depend on any assumption about the distribution of galaxies. To this end, it is proposed to consider in each interval of values of absolute stellar magnitudes $[M, M+d M]$ the ratio of the differential LF of galaxies to the number of galaxies brighter than M. This ratio does not depend on the density distribution of galaxies (it is reduced). In [21] and [22], instead of equal intervals of stellar magnitudes equal intervals of radial velocities were used.

Another method, independent of the distribution of galaxies, which is based on the maximum likelihood method, was proposed in [23] (STY). This method also makes it possible to introduce an amendment due to the incompleteness of galaxies. Unlike the previous ones, this method assumes that the distribution of luminosities is described by an analytical function.

In order not to make any assumptions for the functional approximation of the LF, in [24] a step-bystep maximum likelihood method (SWML) was derived. Galaxies are calculated in apparent magnitude intervals, but no functional form of LF is assumed.

The following method, insensitive to grouping, is based on the maximum likelihood method was proposed in [25]. In this method it is assumed that the distribution of objects is described by a probability distribution of random Poisson processes. Method [25] is also not parametric, but requires splitting data into intervals. The author applied his method to study the sample extracted from the CfAl survey of redshifts [26].

A comparative analysis of these methods has been made in various papers ([22, 24, 25, 27, 28]). The main objective of these studies was to investigate how well the various variants measure the LF of galaxy samples limited by apparent magnitude. In particular, in [27], a comparison of various methods for estimating the LF of galaxies by Monte Carlo methods is presented. The simulation showed that the method [15] and the method STY [23] are the best evaluators of LF. Simulations in [27] also showed that the STY method with a steep slope of the Schechter function tends to lower the slope of the weak end of the LF, which becomes very significant when the sample contains several hundred objects.

The author of [27] believes that, in general, when parameterizing by the Schechter function, the $C^{-}$ 
method is the most stable method relative to the slope of the weak end and the sample size. In his opinion, the $1 / V_{m}$ method does not give reliable results even with a uniform distribution of galaxies in space.

Simulation is also used to compare different estimates of LF normalization. It also shows that most methods tend to restore density by about $20 \%$ lower than the input value.

In order to evaluate the methods for constructing the LF of galaxies, the Monte-Carlo method was also used in [28]. The authors noted four non-parametric methods. These are the $1 / V_{m} \operatorname{method}[13]$, the step-like maximum likelihood method [24] (SWML), the Choloniewski method [25] and the improved Linden-Bell method (see [28]).

In contrast to [27], they showed that the $1 / V_{m}$ method gives absolutely correct results when there is no non-homogeneity, but the results are not reliable in the presence of groupings and voids. They believe that the three other maximum likelihood methods provide fairly reliable and consistent results.

In work [28], it is proposed in practice to use the method [25] for two reasons: first, it immediately gives both form and normalization of the LF, secondly, it is the most quickly calculable from the four methods, due to the simplicity of its algorithm.

In our opinion, the $1 / V_{m}$ method is the simplest of the existing methods. As noted in [27], probably for this reason, despite its shortcomings, this is the most frequently used method (for example, [29, 30]). As noted above, in this paper, this method is generalized in order to apply it to non-uniformly distributed objects [6]. After this, the method remains the simplest method for building the LF of galaxies.

Therefore, it makes sense to compare the proposed method of constructing the LF of galaxies with other methods. This paper presents the LF of galaxies by CfA1 survey, which was compared with the LF of galaxies by CfA2 survey, as well as with LF obtained by other methods, based on the CfA1 catalog provided in [27].

CfA1 is limited in radial velocity $(500 \mathrm{~km} / \mathrm{s} \leqslant V \leqslant 8000 \mathrm{~km} / \mathrm{s})$, and CfA2 is limited in radial velocity $(1000 \mathrm{~km} / \mathrm{s} \leqslant V \leqslant 15000 \mathrm{~km} / \mathrm{s})$, and both surveys are in galactic latitudie $\left(|b I I| \geq 20^{\circ}\right)$.

The absolute magnitudes are calculated by making the appropriate corrections taking into account the galactic absorption [31] and the K-correction, according to the relation $K=k z[24]$.

The heliocentric radial velocities of galaxies were corrected taking into account the movement of the Sun in the Local System, and the movement of the Local system of galaxies in the direction of the cluster in Virgo according to [31].

LF is determined by the method proposed in [6], which is further designated as the method $1 / D_{m} V_{m}$.

Figure 1 shows the normalized LF of CfA1 galaxies obtained using the $1 / D_{m} V_{m}$ method and the $1 / V_{m}$ method [13]. The error bars $1 \sigma$ shown in the figure are defined as follows:

$$
\sigma\left(\Psi\left(M_{i}\right)\right)=\frac{\Psi\left(M_{i}\right)}{n_{i}}\left[n_{i}\left(1-\frac{n_{i}}{N}\right)\right]^{1 / 2}
$$

The well-known result that the $1 / V_{m}$ method overestimates the relative numbrer of faint galaxies is clearly visible from the figure.

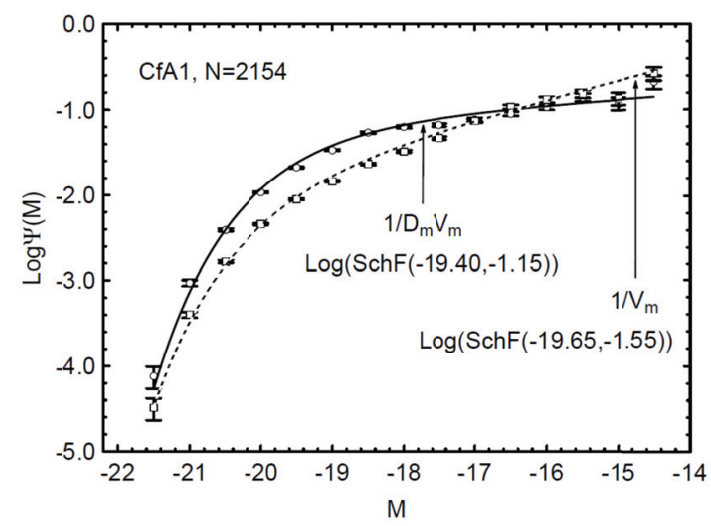

Figure 1. The normalized LF of the CfA1 survey of galaxies, according to the method proposed by us $\left(1 / D_{m} V_{m}\right)$ and by the method $1 / V_{m}[13]$. 
Figure 2 shows the LF of galaxies of the CfA1 and CfA2 surveys, constructed by the method $1 / D_{m} V_{m}$. As can be seen from the figure, the depth of sampling does not affect the results.

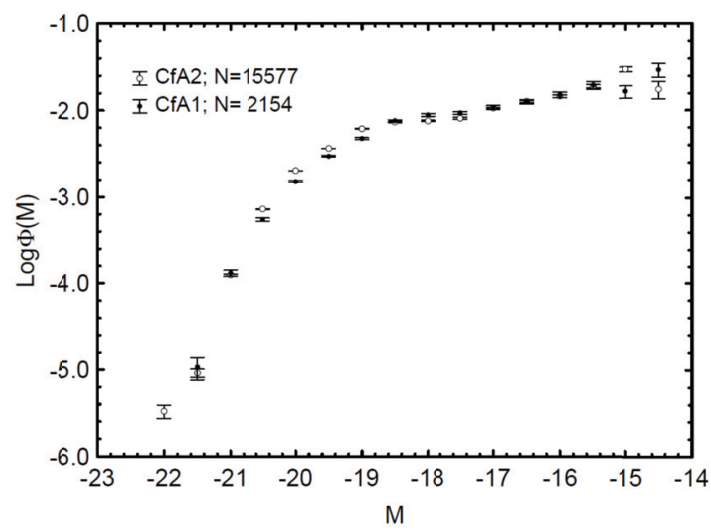

Figure 2. LF of galaxies of surveys CfA1 and CfA2 constructed by the methord $1 / D_{m} V_{m}$.

In Table 1. the parameters $\alpha$ and $M^{*}$ are given in representations by the Schechter function [33] of the CfA1 galaxy LF of the redshift survey obtained by different methods. The results of the first six lines are borrowed from [27]. The last two are our results from our method $1 / D_{m} V_{m}$ and the Schmidt method $1 / V_{m}$. The results of Table 1 are graphically compared in Fig. 3. On the graph, the curves are arbitrarily moved along the ordinate axis. From the figure it is clear that all the methods described, except for the method $1 / V_{m}$ give similar results.

Thus, the proposed method in this paper $1 / D_{m} V_{m}$ to build a LF of galaxies, gives a result consistent with other methods, is not inferior in accuracy to other methods, and is the simplest.

Table 1. Parameters $\alpha$ and $M^{*}$ in the representations by the function of the shehter LF galaxy CfA1 survey of red shifts obtained by different methods

\begin{tabular}{cccc}
\hline Method & $\alpha$ & $M^{*}$ & note \\
\hline SWML & $-1.20 \pm 0.03$ & $-19.30 \pm 0.04$ & $\Delta M=0.25$ \\
STY & $-1.11 \pm 0.08$ & $-19.17 \pm 0.08$ & \\
Choloniewski & $-1.18 \pm 0.05$ & $-19.26 \pm 0.07$ & $\Delta M=0.25$ \\
Turner & $-1.11 \pm 0.06$ & $-19.32 \pm 0.05$ & $\Delta z=500 \mathrm{~km} / \mathrm{s}$ \\
$1 / V_{m}, \mathrm{~W}$ & $-1.59 \pm 0.04$ & $-19.43 \pm 0.07$ & $\Delta M=0.25$ \\
$C^{-}$ & $-1.20 \pm 0.01$ & $-19.21 \pm 0.01$ & \\
$1 / D_{m} V_{m}$ & $-1.15 \pm 0.04$ & $-19.40 \pm 0.05$ & $\Delta M=0.20$ \\
$1 / V_{m}$ & $-1.55 \pm 0.01$ & $-19.65 \pm 0.01$ & $\Delta M=0.20$ \\
\hline
\end{tabular}

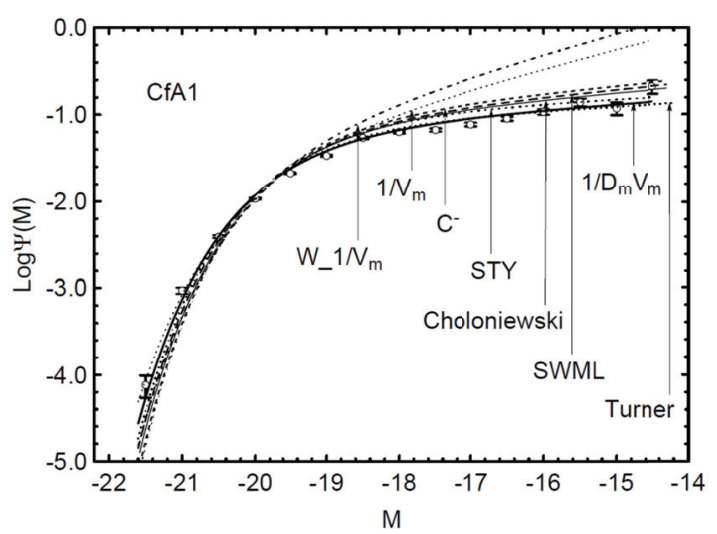

Figure 3. The CfA1 galaxy LF of the redshift survey obtained by different methods: Curves are arbitrarily moved along the ordinate axis. 


\section{Method of Identifying Groups of Galaxies}

To identify groups of galaxies, the method proposed in [34, 35] was used.

According to this method, an arbitrary galaxy is first selected from a list bounded by the apparent magnitude and with full radial velocity data, and then neighbors are searched for in its vicinity by the following criteria:

$$
\begin{gathered}
\frac{\max \left(k_{T_{1}} L_{1}, k_{T_{2}} L_{2}\right)}{D_{12}} \geq E_{0} \\
\left|V_{1}-V_{2}\right| \leq V_{0}
\end{gathered}
$$

where $k_{T_{1}}$ and $k_{T_{2}}$ - are coefficients depending on the morphological types of galaxies: $k_{T_{i}}=f\left(T_{i}\right) / f(S)$, $f\left(T_{i}\right)$ - is the mass to luminosity ratio $\left(\right.$ Mass $/ L$ ) for a given morphological type $T_{i}$, and $f(S)$ - is the similar value for spiral galaxies (i.e. $k_{S}=1$ ). $V_{1}$ and $V_{2}$ are the radial velocities of the first and second galaxies, respectively, $L_{1}$ and $L_{2}$ are their luminosities, $D_{12}$ - is the linear distance in the projection on the celestial sphere. $E_{0}$ and $V_{0}$ are free parameters whose values have to be chosen.

In $[34,35]$, we showed that when using the neighborhood criterion (7) and (8), there is no need to change the parameters $E_{0}$ and $V_{0}$ with distance (in order to take into account changes in the observed luminosity function of galaxies). At the same time, both nearby and distant groups are identified with equal success.

To determine parameter values $E_{0}$ and $V_{0}$, in which the identified groups will be physically real systems, lists of groups are compiled for the whole grid of parameter values $E_{0}$ and $V_{0}$. The dependences of the average characteristics of the identified groups (average velocity dispersions of galaxies in the groups, the average mass-to-luminosity ratio, the number of systems detected) on the value of $E_{0}$ and $V_{0}$ are studied. Intervals $E_{0}^{(1)} \leq E_{0} \leq E_{0}^{(2)}$ and $V_{0}^{(1)} \leq V_{0} \leq V_{0}^{(2)}$ were selected, where the average characteristics of groups are relatively weakly dependent on $E_{0}$ and $V_{0}$, compared to outside this area.

It is assumed that the groups identified by randomly selected pairs $E_{0}$ and $V_{0}$ of these areas will be practically real physical systems.

\section{Sample}

We use the same sample of groups and ArG as in [4]. The groups of galaxies are used as groups [35], identified on the basis of the CfA2 redshift survey (http://vizier.cfa.harvard.edu/viz-bin/VizieR?source $=\mathrm{VII} / 256)$.

Considering that Hubble's law has a large uncertainty at nearby distances, in identifying groups of galaxies, we limited the CfA2 sample in terms of radial velocities to below: $V \geq 1000 \mathrm{~km} / \mathrm{s}$.

Also, bearing in mind that at large distances the completeness of the CfA2 catalog is rather small, we have limited the sample to the radial velocities above: $V \leq 15000 \mathrm{~km} / \mathrm{s}$.

To reduce the effect of light attenuation in the galaxy, a restriction on the galactic latitude was also introduced: $|b I I| \geq 20^{\circ}$. As a result, out of 18203 CfA2 survey galaxies, our sample included 15577 galaxies (172 of which are ArG), which were used to create a list of groups of galaxies and single galaxies.

In the identified 1971 groups with 2 or more members, 6787 galaxies were included, 2412 of which are members of double galaxy systems. The remaining 8790 galaxies make up a sample of "single" galaxies.

The number of ArGs among single galaxies is 92 galaxies. The number of ArGs in the groups is 80 galaxies. Morphological types of 135 ArG are known, 65 of which are included in the group.

\section{The Luminosity Function of Arakelian Galaxies}

Figure 4 shows the normalized luminosity functions for the galaxies of the entire CfA2 survey sample and the ArGs included in this sample. If the galaxy is a member of the system, then its distance is 
estimated from the radial velocity of the system. LF are represented by the Schechter function [33].

In Fig. 4, it can be seen that in the bright and weak regions, the normalized LF of Arakelian galaxies is higher than the analogous function of the total sample CfA2. A similar phenomenon is observed for group member galaxies, as well as for single galaxies (Fig. 5).
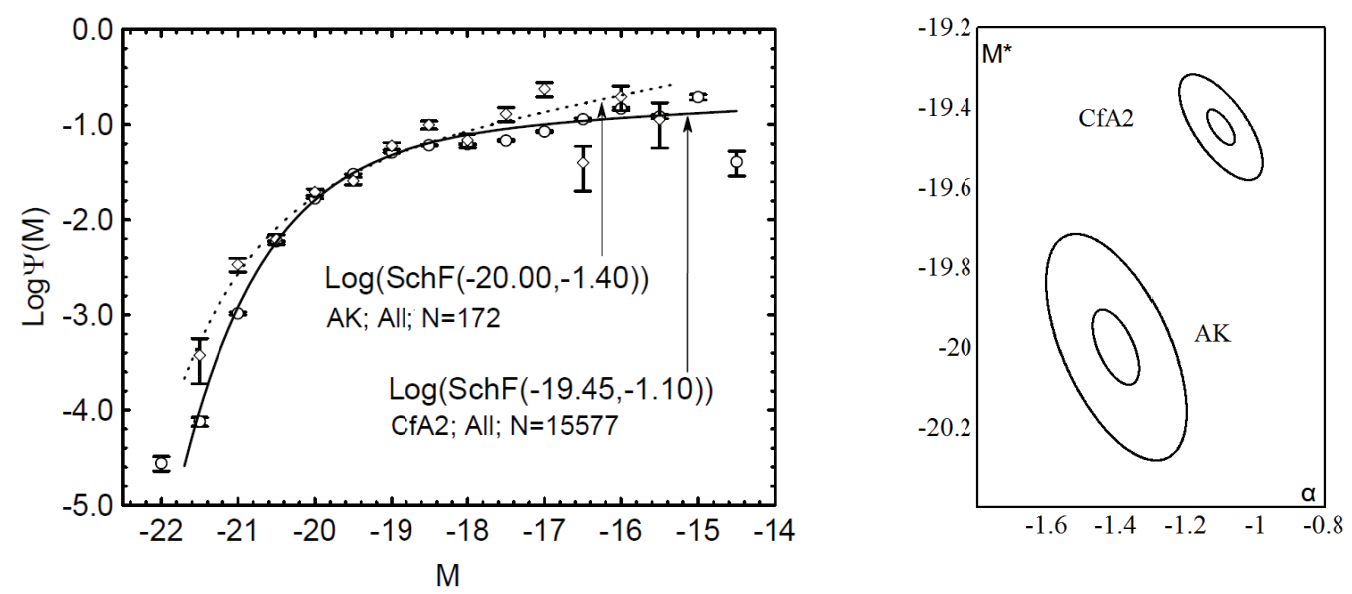

Figure 4. Normalized LF of the entire sample of CfA2 and ArG. The right side shows the corresponding error ellipses of the parameters $M^{*}$ and $\alpha$ in the approximation of the Schechter function (accordingly, for $1 \sigma$ and $3 \sigma$ ).
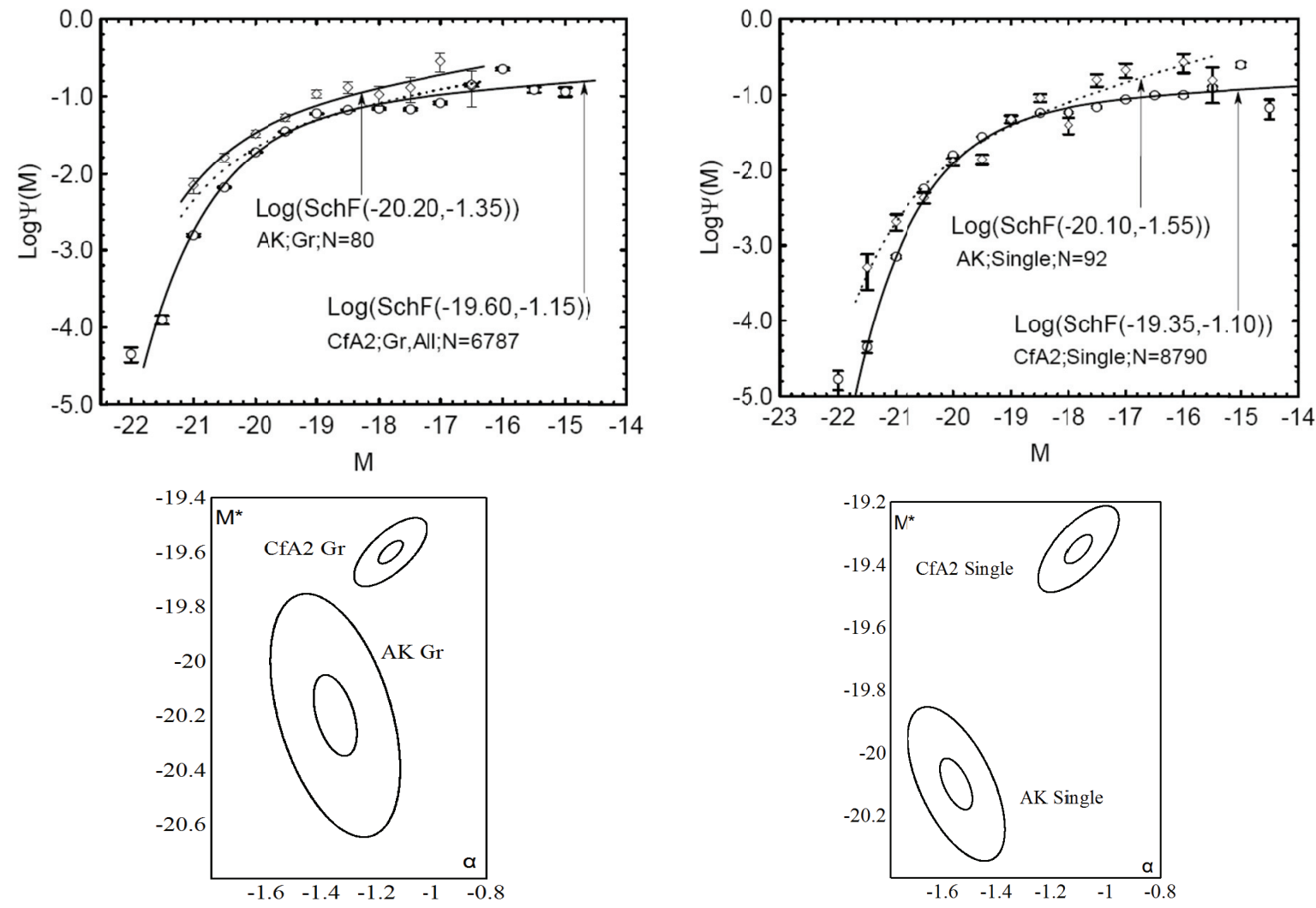

Figure 5. The normalized LF of members of the CfA2 and ArG groups belonging to these groups (left), and of single CfA2 galaxies and ArG. The dashed line on the left side is the Schechter function of the ArGLF arbitrarily moved along the ordinate axis. Below are the corresponding error ellipses of the parameters $M^{*}$ and $\alpha$ with approximation by Schechter's function (accordingly, for $1 \sigma$ and $3 \sigma$ ). 

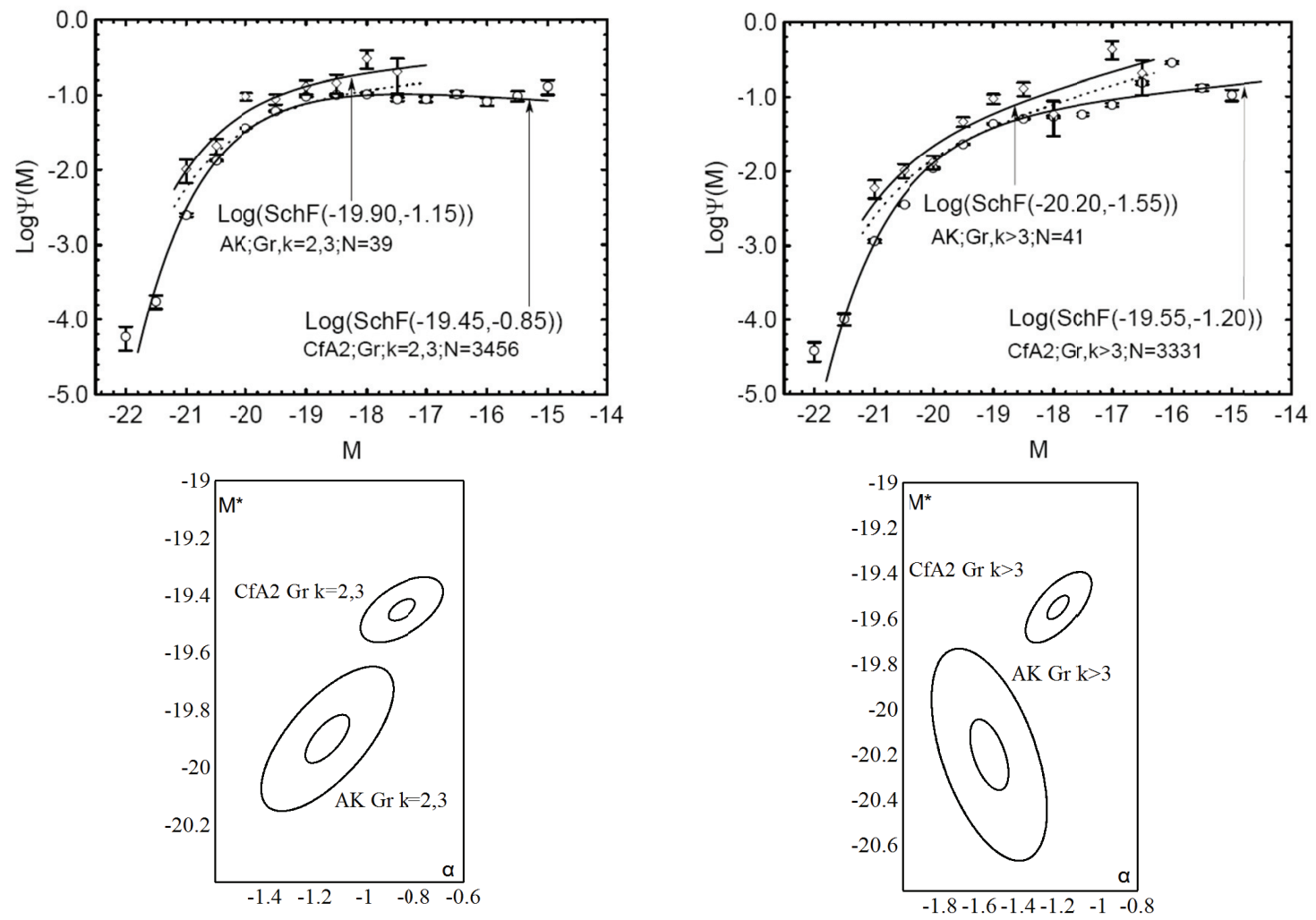

Figure 6. The normalized LF of members of the CfA2 groups, having in its composition 2 or 3 galaxies and ArG, included in these groups (left figure), and for groups having more than 3 galaxies. The dashed lines - are Schechter's function of the ArG LF arbitrarily moved along the ordinate axis. Below are the corresponding error ellipses of the parameters $M^{*}$ and $\alpha$ with approximation by Schechter's function (accordingly, for $1 \sigma$ and $3 \sigma$ ).

Figure 6 presents the normalized LF of members of the CfA2 groups, having in their composition 2 or 3 galaxies and the ArG included in these groups, and for groups having more than 3 galaxies. The dotted lines are Schechter's function of the ArG LF arbitrarily moved. It can be seen from the figure that the above also holds when we consider small and relatively populated groups separately.

In Fig. 7. The normalized LF of elliptical and lenticular galaxies and spiral and irregular CfA2 and ArG galaxies are included in this survey. It can be seen from the figure that spiral and irregular galaxies repeat the above result. But for elliptical and lenticular galaxies, due to the small number of objects, it is impossible to say anything. In the range of absolute magnitudes $M<-19.5$, we have a total of 8 objects.
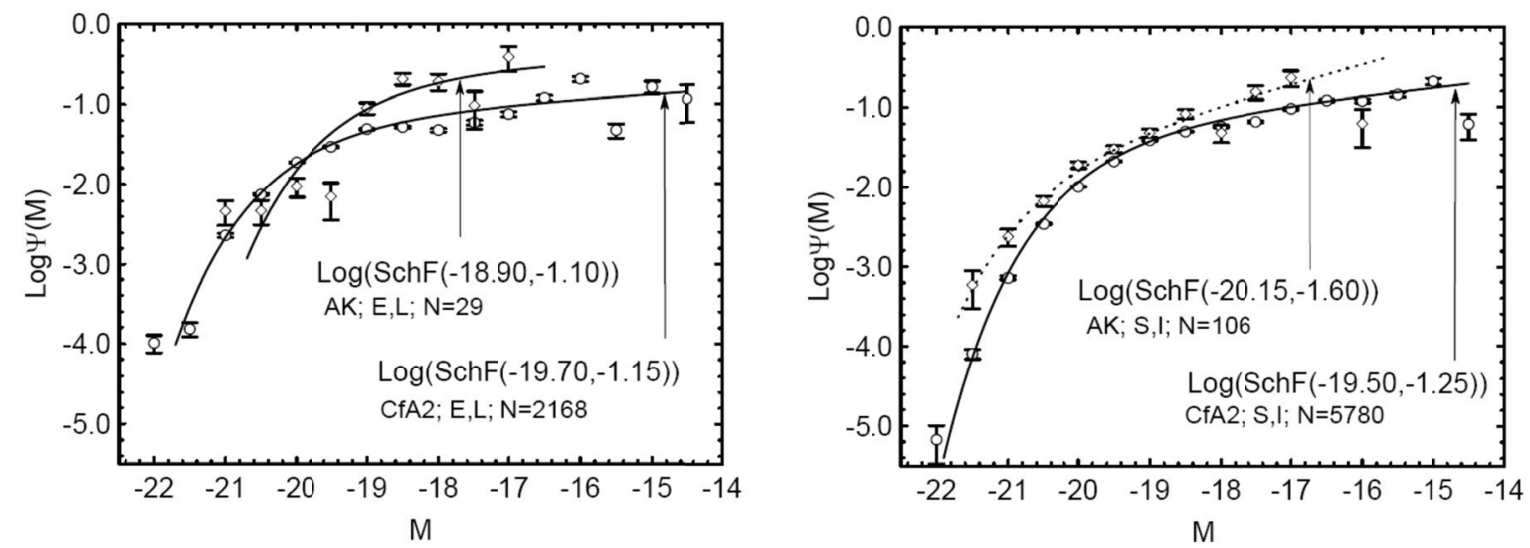

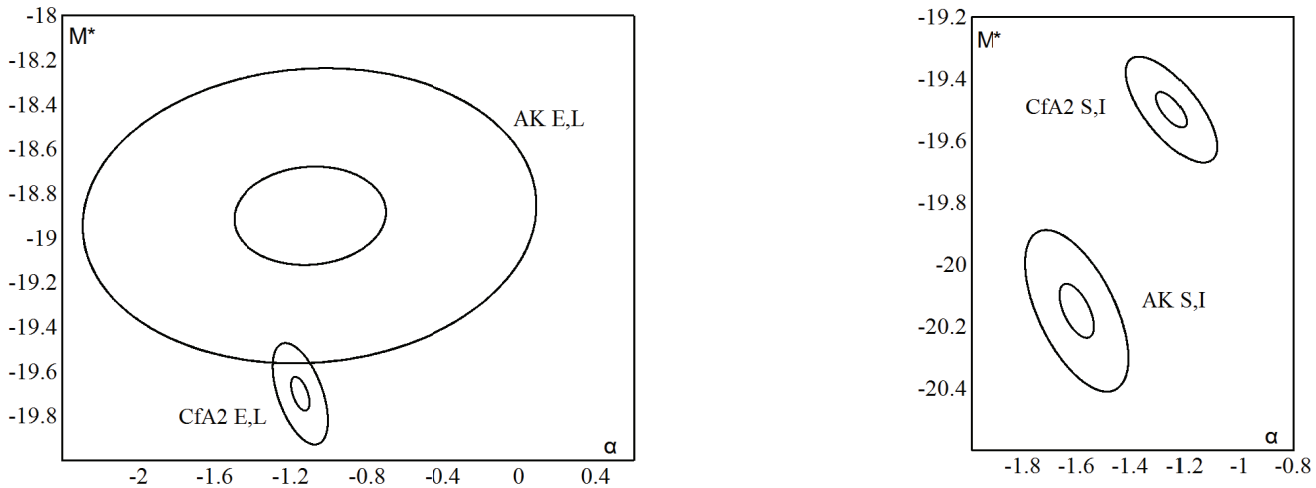

Figure 7. The normalized LF of elliptical and lenticular galaxies (left figure), spiral, and irregular galaxies from the CfA2 survey, and ArG of the corresponding morphological types included in this survey. Below are the corresponding error ellipses of the parameters $M^{*}$ and $\alpha$ with approximation by Schechter"s function (accordingly, for $1 \sigma$ and $3 \sigma)$.
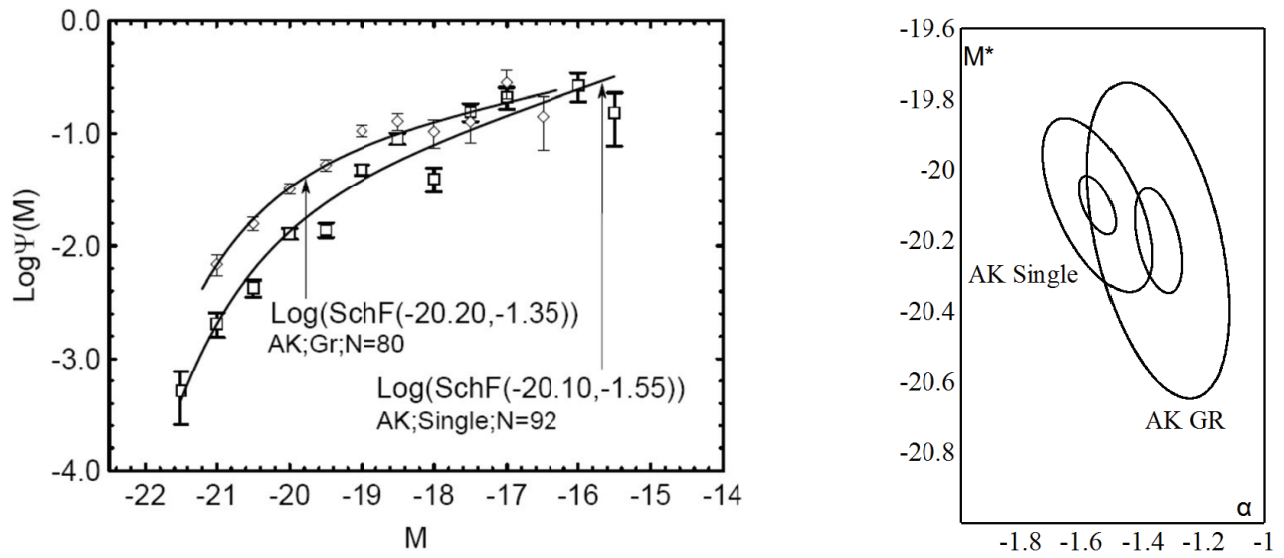

Figure 8. Normalized LF of members of groups and single ArG. The right side shows the corresponding error ellipses of the parameters $M^{*}$ and $\alpha$ for approximation by the Schechter function (for $1 \sigma$ and $3 \sigma$, respectively).

Figure 8 presents the normalized LF of group members and single ArG. As can be seen from the figure of the LF of the ArG, the members of groups and single ArG do not differ much. It is possible that there are more bright ArG in groups than among Arakelian's single galaxies. Recall that in [4] we obtained that ArG in groups, on average, have higher luminosities than single ArG.

Thus, among Arakelian galaxies, there is a deficit of medium luminosity galaxies (about $M=-19 ; H$ $=100 \mathrm{~km} / \mathrm{s} / \mathrm{Mpc}$ ). This fact is valid for both small and relatively populated groups, as well as for single galaxies.

\section{Spatial Density of ArG}

From the above graphs it can be seen that in the sample we used there are no data on the ArG of low luminosities. Therefore, for comparison, we present the spatial densities of the number of galaxies of different subsamples CfA2 and ArG in the range of absolute values $M \leqslant-17$.

Table 2 for galaxies in the range of absolute values $M \leqslant-17$ shows the spatial density of the number of galaxies of the CfA2 survey and ArG in different subsamples and the corresponding percentage of ArG in this subsample. It can be seen that the relative amount of ArG among elliptical and lenticular galaxies is somewhat higher, which is consistent with [4].

For the entire ArG sample, one can estimate the number density up to $M=-15.5 . \rho(M \leqslant-$

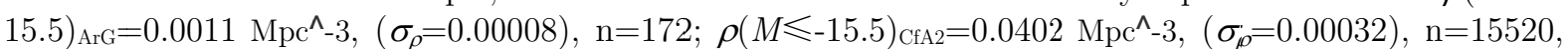


so among the galaxies with $M \leqslant-15.52 .6 \%$ are ArG. This is slightly higher than the similar value obtained in [4] (2.05\%). This is due to the fact that in [4] the samples are limited by the apparent magnitude and do not take into account the distribution of absolute stellar magnitudes.

Table 2. Spatial density of the number of galaxies of the CfA2 survey and arg in different selections

\begin{tabular}{|c|c|c|c|c|c|}
\hline Sample & & $\mathbf{n}$ & $\rho(M \leqslant-17)$ & $\sigma_{\rho}$ & $\%$ ArG \\
\hline \multirow{2}{*}{ All galaxies } & CfA2 & 14871 & 0.02542 & 0.00021 & \multirow{2}{*}{$2.7 \%$} \\
\hline & ArG & 167 & 0.00068 & 0.00005 & \\
\hline \multirow{2}{*}{ Single galaxies } & CfA2 & 8419 & 0.01459 & 0.00016 & \multirow{2}{*}{$2.8 \%$} \\
\hline & ArG & 88 & 0.00041 & 0.00004 & \\
\hline \multirow{2}{*}{ Members of galaxy groups } & CfA2 & 6452 & 0.01087 & 0.00014 & \multirow{2}{*}{$2.6 \%$} \\
\hline & ArG & 79 & 0.00028 & 0.00003 & \\
\hline \multirow{2}{*}{ Group members with $\mathrm{k}=2,3$} & CfA2 & 3394 & 0.00477 & 0.00008 & \multirow{2}{*}{$(1.7 \%)^{*}$} \\
\hline & ArG & 39 & $(0.00008)^{*}$ & 0.00001 & \\
\hline \multirow{2}{*}{ Group members with $\mathrm{k}>3$} & CfA2 & 3058 & 0.00611 & 0.00012 & \multirow{2}{*}{$3.4 \%$} \\
\hline & $\mathrm{ArG}$ & 40 & 0.00021 & 0.00003 & \\
\hline \multirow{2}{*}{$\mathrm{E}, \mathrm{L}$} & CfA2 & 2067 & 0.00476 & 0.00011 & \multirow{2}{*}{$4.0 \%$} \\
\hline & ArG & 29 & 0.00019 & 0.00004 & \\
\hline \multirow{2}{*}{ S,I } & CfA2 & 6305 & 0.01659 & 0.00023 & \multirow{2}{*}{$3.0 \%$} \\
\hline & $\mathrm{ArG}$ & 102 & 0.00049 & 0.00005 & \\
\hline
\end{tabular}

* - this is the lower limit $(\rho=0.00008$ corresponds for $M \leqslant-17.5)$

\section{Conclusion}

We have proposed a method for constructing the LF of galaxies, which generalizes the $1 / V_{m}$ method of Schmitd [7], taking into account the uneven distribution of galaxies in the nearby Universe [7]. We call it the $1 / D_{m} V_{m}$ method. In this paper, we compare the proposed method for constructing the LF of galaxies with some other known methods that are also independent of the spatial distribution of galaxies. They are known in the literature as SWML methods [24], STY [23], Choloniewski [25], Turner [19]. Our comparison shows that the method $1 / D_{m} V_{m}$ gives a result consistent with other methods.

A comparison is also made of the LF of galaxies of the CfA1 and CfA2 redshift surveys obtained by the $1 / D_{m} V_{m}$. They almost coincide. This means that the function $D(r)$ in [6] is defined quite reliably.

The luminosity functions of different morphological types belonging to the CfA2 catalog of the Arakelian galaxies in different environments are constructed.

It was found that both the bright and weak parts of the normalized luminosity function of Arakelian galaxies are higher than the bright and weak parts of the analogous function of the total sample CfA2. A similar phenomenon is observed separately for members of groups, as well as for single galaxies. This also applies to small groups with 2 or 3 galaxies, and for more populated groups. Spiral galaxies obey these laws. For elliptical galaxies, the picture is not very clear (due to the small number of known elliptical galaxies of Arakelian). This means that the galaxies of Arakelian have a shortage of mediumluminosity galaxies (about $M=-19 ; H=100 \mathrm{~km} / \mathrm{s} / \mathrm{Mpc}$ ).

Among the galaxies with $M \leqslant-15.5,2.6 \%$ are Arakelian galaxies with high surface brightness.

Acknowledgements. On the 90th anniversary of M. A. Arakelian.

\section{References}

1. M. A. Arakelian, Soobshch. BAO, 47, 3 (1975).

2. F. Zwicky, E. Herzog, P. Wild, M. Karpowicz, and C. Kowal, Catalogue of Galaxies and Clusters of Galaxies, Vols. 1-6 (1961-1968).

3. B. Vorontsov-Vel'yaminov, A. Krasnogorskaya, and V. Arkhipova, Morphological Catalog of Galaxies, Vols. 1-3 (1962-1964). 
4. A.P.Mahtessian, V.G. Movsessian, Astrophysics, 53, 189, 2010.

5. V.K. Golev, Z.I. Tsvetanov, G.T. Petrov, Compesrendus de l'Academiebulgare des Sciences, 37, 15, 1984.

6. A.P.Mahtessian, Astrophysics, 54, 189, 2011.

7. M.Schmidt, Astrophys. J., 151, 393, 1968.

8. J.Neyman, E.L.Scott, Confrontation of Cosmological Theories with Observational Data, IAUS No. 63, Ed. by Longair, 129, 1974.

9. V.Yu.Terebizh, Astrophysics, 16, 45, 1980.

10. I.K. Baldry, S.P. Driver, J. Loveday, E.N. Taylor, L.S. Kelvin, J. Liske, P. Norberg, A.S.G. Robotham, S. Brough, A.M. Hopkins, S.P. Bamford, J.A. Peacock, J. Bland-Hawthorn, C.J. Conselice, S.M. Croom, H.R. Parkinson, C.C. Popescu, M. Prescott, R.G. Sharp, R.J. Tuffs, Mon. Notic. Roy. Astron. Soc., 421, 621, 2012.

11. J.E. Felten, Astron. J., 82, 861, 1977.

12. B. Binggeli, A. Sandage, G.A.Tammann, Ann. Rev. Astron. Astrophys., 26, 509, 1988.

13. M. Schmidt, Astrophys. J., 151, 393, 1968.

14. J.P. Huchra, W.L.W. Sargent, Astrophys. J., 186, 433, 1973.

15. D. Lynden-Bell, Mon. Notic. Roy. Astron. Soc., 155, 95, 1971.

16. J.C. Jackson, Mon. Notic. Roy. Astron. Soc., 166, 281, 1974.

17. J.F. Nicoll, I.E. Segal, Astron. Astrophys., 118, 180, 1983.

18. J. Choloniewski, Mon. Notic. Roy. Astron. Soc., 226, 273, 1987.

19. E.L. Turner, Astrophys. J., 231, 645, 1979.

20. R.P. Kirshner, A. Oemler, P.L. Schlechter, Astron. J., 84, 951, 1979.

21. M. Davis, J. Huchra, Astrophys. J., 254, 437, 1982.

22. V. de Lapparent, M.J. Geller, J.H. Huchra, Astrophys. J., 343, 1, 1989.

23. A. Sandage, G.A. Tammann, A. Yahil, Astrophys. J., 232, 352, 1979.

24. G. Efstathiou, R.S. Ellis, B.A. Peterson, Mon. Notic. Roy. Astron. Soc., 232, 431, 1988.

25. J. Choloniewski, Mon. Notic. Roy. Astron. Soc., 223, 1, 1986.

26. J.P. Huchra, M. Davis, D. Latham, J. Tonry, Astrophys. J. Suppl. Ser., 52, 89, 1983.

27. C.N.A. Willmer, Astron. J., 114, 898, 1997.

28. T.T. Takeuchi, K. Yoshikawa, T.T. Ishii, Astrophys. J. Suppl. Ser., 129, 1, 2000.

29. S.J. Lilly, L. Tresse, F. Hammer, D. Crampton, O. Le Fevre, Astrophys. J., 455, 108, 1995.

30. R.S. Ellis, M. Colless, T. Broadhurst, J. Heyl, K. Glazebrook, Mon. Notic. Roy. Astron. Soc., 280, 235, 1996.

31. A. Sandage, Astrophys. J., 183, 711, 1973.

32. R. Nolthenius, S.D.M. White, Mon. Notic. Roy. Astron. Soc., 235, 505, 1987.

33. P. Schechter, 1976, ApJ, 203, 297.

34. A.P.Mahtessian, Astrophysics, 28, 255, 1988.

35. A.P.Mahtessian, V.G. Movsessian, Astrophysics, 53, 83, 2010. 